\title{
VIII. Ventilación no invasiva en la desvinculación de la ventilación mecánica
}

\author{
FERNANDO SALDÍAS P.* y FRANCISCO ARANCIBIA H.**
}

\author{
VIII. Noninvasive ventilation in the weaning of patients with respiratory failure
}

La intubación endotraqueal y la ventilación mecánica (VM) se asocian a graves complicaciones, tales como daño de la vía aérea superior, barotrauma e infecciones, especialmente neumonía nosocomial que se asocia a riesgo de muerte elevado ${ }^{1}$. La desconexión de VM dificultosa o weaning prolongado se asocia a mayor riesgo de neumonía nosocomial, aumento de la morbimortalidad, especialmente en pacientes con insuficiencia respiratoria crónica $^{2,3}$. Acortar el tiempo de intubación y conexión a ventilación mecánica constituye un desafío para los clínicos, que nos obliga a considerar el mejor momento para iniciar el proceso de desconexión del ventilador, minimizando los riesgos de fracaso y reintubación. La reintubación después de una desconexión fallida del ventilador constituye un factor de riesgo independiente de neumonía nosocomial, estadía prolongada en el hospital y letalidad elevada ${ }^{4}$. En este contexto clínico, los principales objetivos de la ventilación no invasiva (VNI) son mejorar la oxigenación y la ventilación de los pacientes con insuficiencia respiratoria parcial o global, evitando la reintubación y conexión a ventilador mecánico, reduciendo los riesgos y complicaciones asociados ${ }^{5}$. Revisaremos el papel de la VNI como herramienta de apoyo durante el proceso de desconexión del ventilador mecánico, incluyendo el destete difícil y la falla respiratoria aguda post extubación.

Pregunta: ¿La ventilación no invasiva reduce el riesgo de reintubación, complicaciones y muerte aplicada como medida de apoyo durante el proceso de desconexión del ventilador?.

Pacientes: Sujetos ventilados por falla respiratoria aguda que requieren asistencia ventilatoria artificial durante el período de desconexión del ventilador mecánico.
Intervención: VNI aplicada durante el período de desconexión del ventilador.

Objetivo: Evaluar si la VNI reduce el riesgo de reintubación, complicaciones y muerte aplicada a los pacientes durante el proceso de desconexión del ventilador mecánico.

\section{Resumen de la evidencia}

La VNI se ha empleado en tres escenarios clínicos distintos en la desconexión de ventilación mecánica: a) La VNI facilitaría la desconexión del ventilador; b) La VNI disminuiría el riesgo de fracaso de la extubación en pacientes con riesgo elevado; y c) La VNI permitiría reducir el riesgo de fracaso de la extubación en pacientes con falla respiratoria progresiva post extubación ${ }^{6,7}$.

a) Facilitar la desconexión de VM. En la mayoría de los casos, el retiro de la ventilación mecánica es posible inmediatamente después que se resuelve el problema que ocasionó la falla respiratoria aguda. Sin embargo, existe un grupo de pacientes ventilados que requiere un retiro más gradual o prolongado del ventilador. Varios estudios recientes han investigado el papel de la VNI para facilitar la extubación y desconexión del ventilador (Tabla 1$)^{8-12}$. La VNI se ha empleado principalmente en pacientes con EPOC, después del fracaso de una o varias pruebas de tubo $\mathrm{T}^{8-10}$. Nava y cols, evaluaron la eficacia de la VNI en el proceso de desconexión de VM en pacientes con EPOC exacerbado y prueba de tubo $\mathrm{T}$ fallida a las 48 horas $^{8}$. En 50 pacientes con desconexión fallida se evaluó la eficacia de la VNI por máscara facial comparado con la VM convencional con presión de soporte. En los pacientes manejados con VNI, la permanencia en VM $(10,2 \pm 6,8$ vs 16,6 $\pm 11,8$ días;

\footnotetext{
* Profesor Asociado de Medicina, Departamento de Enfermedades Respiratorias, Pontificia Universidad Católica de Chile.

** Unidad de Cuidados Intensivos, Instituto Nacional del Tórax, Santiago, Chile.
} 
Tabla 1. Estudios clínicos controlados que examinan la eficacia de la ventilación no invasiva en pacientes con destete difícil del ventilador mecánico

\begin{tabular}{|c|c|c|c|c|}
\hline Efecto de VNI & Nava $^{8}$ & Girault $^{9}$ & Chen $^{10}$ & Ferrer $^{11}$ \\
\hline Publicación & 1998 & 1999 & 2001 & 2003 \\
\hline Modalidad & VNI-PS & VNI-P/F & VNI-PS & BiPAP \\
\hline Interface & Máscara facial & Máscara facial o nasal & Máscara facial & Máscara facial o nasal \\
\hline Pacientes & $\begin{array}{l}50 \text { pacientes con } \\
\text { EPOC exacerbado, } \\
\text { falla respiratoria hi- } \\
\text { percápnica y destete } \\
\text { fallido a las } 48 \text { horas }\end{array}$ & $\begin{array}{l}33 \text { pacientes con falla } \\
\text { respiratoria aguda } \\
\text { sobre crónica y } \\
\text { destete fallido a las } \\
48 \text { horas }\end{array}$ & $\begin{array}{l}24 \text { pacientes con } \\
\text { EPOC exacerbado y } \\
\text { destete a las } 72 \text { horas }\end{array}$ & $\begin{array}{l}43 \text { pacientes con falla } \\
\text { respiratoria aguda y } \\
\text { destete fallido durante } \\
\text { tres días consecutivos }\end{array}$ \\
\hline Intervención & $\begin{array}{l}\text { VNI: } 25 \\
\text { VM: } 25\end{array}$ & $\begin{array}{l}\text { VNI: } 17 \\
\text { VM: } 16\end{array}$ & $\begin{array}{l}\text { VNI: } 12 \\
\text { VM: } 12\end{array}$ & $\begin{array}{l}\text { VNI: } 21 \\
\text { VM: } 22\end{array}$ \\
\hline $\begin{array}{l}\text { Incidencia de } \\
\text { neumonía }\end{array}$ & $\begin{array}{l}\text { VNI: } 0 \% \\
\text { VM: } 28 \% \\
\text { p }<0,05\end{array}$ & $\begin{array}{l}\text { VNI: } 5,8 \% \\
\text { VM: } 6,2 \% \\
\text { NS }\end{array}$ & $\begin{array}{l}\text { VNI: } 0 \% \\
\text { VM: } 58 \% \\
p=0,027\end{array}$ & $\begin{array}{l}\text { VNI: } 24 \% \\
\text { VM: } 59 \% \\
p=0,042\end{array}$ \\
\hline Estadía en UCI & $\begin{array}{l}\text { VNI: } 15,1 \pm 5,4 d \\
\text { VM: } 24,0 \pm 13,7 \\
p=0,005\end{array}$ & $\begin{array}{l}\text { VNI: } 12,3 \pm 6,8 \mathrm{~d} \\
\text { VM: } 14,0 \pm 7,5 \mathrm{~d} \\
\text { NS }\end{array}$ & & $\begin{array}{l}\text { VNI: } 14,1 \pm 9,2 \mathrm{~d} \\
\text { VM: } 25,0 \pm 12,5 \mathrm{~d} \\
\mathrm{p}=0,002\end{array}$ \\
\hline $\begin{array}{l}\text { Estadía en el } \\
\text { hospital }\end{array}$ & & $\begin{array}{l}\text { VNI: } 27,1 \pm 14,3 \mathrm{~d} \\
\text { VM: } 27,6 \pm 13,0 \mathrm{~d} \\
\text { NS }\end{array}$ & $\begin{array}{l}\text { VNI: } 16 \pm 6 \mathrm{~d} \\
\text { VM: } 25 \pm 12 \mathrm{~d} \\
\mathrm{p}<0,05\end{array}$ & $\begin{array}{l}\text { VNI: } 27,8 \pm 14,6 \mathrm{~d} \\
\text { VM: } 40,8 \pm 21,4 \mathrm{~d} \\
\mathrm{p}=0,026\end{array}$ \\
\hline $\begin{array}{l}\text { Sobrevida en el } \\
\text { seguimiento }\end{array}$ & $\begin{array}{l}\text { VNI: } 92 \% \\
\text { VM: } 72 \% \\
p=0,009\end{array}$ & $\begin{array}{l}\text { VNI: } 100 \% \\
\text { VM: } 87,5 \% \\
\text { NS }\end{array}$ & $\begin{array}{l}\text { VNI: } 100 \% \\
\text { VM: } 75 \% \\
\text { NS }\end{array}$ & $\begin{array}{l}\text { VNI: } 71,4 \% \\
\text { VM: } 40,9 \% \\
p=0,018\end{array}$ \\
\hline
\end{tabular}

VNI-PS: ventilación no invasiva-presión de soporte, VNI-P/F: ventilación no invasiva-modos de presión o flujo, BiPAP: presión positiva en la vía aérea de dos niveles. El período de seguimiento fue variable en los estudios: Chen (hospital), Nava (2 meses), Girault y Ferrer (3 meses).

$\mathrm{p}=0,021)$ y en la UCI $(15,1 \pm 5,4$ vs $24,0 \pm$ $13,7$ días; $\mathrm{p}=0,005)$ fueron más breves, y el riesgo de neumonía nosocomial $(0 \%$ vs $28 \%)$ y muerte en el seguimiento a 60 días ( $8 \%$ vs 28\%) fueron significativamente reducidos. Girault y cols, evaluaron la eficacia de la VNI comparado con la VM con presión de soporte en 33 pacientes con falla respiratoria aguda sobre crónica con prueba de tubo $\mathrm{T}$ fallida a las dos horas ${ }^{9}$. Ambos procedimientos mejoraron significativamente la ventilación y el intercambio gaseoso, sin embargo, la estadía en UCI y el hospital, y la sobrevida a los tres meses fueron similares en ambos grupos.

En un ensayo clínico, Chen y cols, evaluaron a 24 pacientes con EPOC exacerbado que fueron ventilados durante tres días y luego fueron asignados aleatoriamente a recibir VNI post extubación o continuar en ventilación mecáni$\mathrm{ca}^{10}$. En el grupo asignado a VNI disminuyó significativamente el riesgo de neumonía nosocomial ( $0 \%$ vs $58 \%$; $\mathrm{p}=0,027)$, el tiempo de permanencia en VM $(7 \pm 5$ vs $15 \pm 12$ días; $\mathrm{p}<0,05)$ y en el hospital $(16 \pm 6$ vs. $25 \pm 12$ días; $\mathrm{p}<0,05)$. En resumen, los pacientes con
EPOC exacerbado que fueron ventilados durante tres días y luego recibieron VNI, tuvieron menor incidencia de neumonía nosocomial, acortando su permanencia en ventilación mecánica y en el hospital.

Ferrer y cols, evaluaron el efecto de la VNI por máscara facial en pacientes con desconexión fallida de VM durante tres días consecutivos, comparado con el manejo estándar, en 43 pacientes ventilados, de los cuales, 33 tenían enfermedad respiratoria crónica ${ }^{11}$. La VNI disminuyó significativamente el tiempo de permanencia en VM $(11,4 \pm 8,0$ vs $20,1 \pm 13,1 ; \mathrm{p}=0,012)$, en la UCI $(14,1 \pm 9,2$ vs $25,0 \pm 12,5 ; \mathrm{p}=0,002)$, y el hospital $(27,8 \pm 14,6$ vs 40,8 $\pm 21,4$; $\mathrm{p}=0,026$ ), el riesgo de neumonía nosocomial $(23,8 \%$ vs $59 \%$; $\mathrm{p}=0,042)$ y sepsis $(9,5 \%$ vs $40,9 \% ; \mathrm{p}=0,045)$, y mejoró la sobrevida en la UCI $(90 \%$ vs $59 \% ; \mathrm{p}=0,045)$ y en el seguimiento a 90 días $(71 \%$ vs $41 \%$; $\mathrm{p}=0,044)$. El efecto favorable de la VNI sólo se observó en el grupo de pacientes con enfermedad respiratoria crónica. Los autores concluyeron que la extubación precoz asociado a la VNI redujo significativamente la duración de la VM, el tiempo de 
permanencia en el hospital y el riesgo de complicaciones infecciosas, mejorando la sobrevida de los enfermos.

En un meta-análisis de cinco estudios randomizados, que incluyó 171 pacientes, el empleo de VNI comparado con estrategias de desconexión estándar disminuyó la mortalidad (RR: 0,41; IC95\%: 0,22-0,76), la incidencia de neumonía asociada al ventilador (RR: 0,28; IC95\%: 0,09-0,85), la permanencia en VM (diferencia media: -7,33 días; IC95\%: -11,45 a -3,22 días), el tiempo de estadía en la UCI (diferencia media: -6,88 días; IC95\%: -12,6 a -1,15 días), y en el hospital (diferencia media: $-7,33$ días; IC95\%: $-14,05$ a $-0,61$ días) ${ }^{13}$. En el análisis de subgrupos, la reducción de la mortalidad fue mayor en los pacientes con EPOC.

De acuerdo a estos hallazgos, los pacientes con enfermedad pulmonar obstructiva crónica intubados por falla respiratoria hipercápnica y que han fracasado en una prueba de respiración espontánea en tubo $\mathrm{T}$, podrían ser considerados candidatos para una prueba de extubación precoz apoyado con VNI. b) Disminuir el riesgo de fracaso de la extubación. Pocos estudios han examinado el efecto preventivo de la VNI en el proceso de desconexión del ventilador (Tabla 2). Epstein y cols, identificaron a un grupo de pacientes cuyas características clínicas en el momento de la extubación permitían predecir un riesgo elevado de fracaso y necesidad de reintubación ${ }^{14}$. Dos ensayos clínicos controlados han examinado la eficacia de la VNI precoz en pacientes considerados de alto riesgo de fracaso después de la extubación ${ }^{15,16}$. En un estudio multicéntrico italiano enrolaron a 97 pacientes consecutivos ventilados durante más de 48 horas, quienes tenían uno o más de los siguientes factores de riesgo: hipercapnia, insuficiencia cardíaca congestiva, tos ineficaz o secreciones bronquiales excesivas, fracaso de una prueba de desconexión del ventilador, presencia de dos o más comorbilidades, u obstrucción de la vía aérea superior ${ }^{15}$. Después de una prueba de respiración espontánea en tubo $\mathrm{T}$ exitosa, 48 pacientes fueron aleatoriamente asignados a la rama de VNI por lo menos durante 8 horas diarias los primeros

Tabla 2. Estudios clínicos controlados que examinan la eficacia de la ventilación no invasiva en pacientes de alto riesgo de fracaso en el destete del ventilador mecánico

\begin{tabular}{|c|c|c|}
\hline Efecto de VNI & Nava $^{15}$ & Ferrer $^{16}$ \\
\hline Publicación & 2005 & 2006 \\
\hline Modalidad & BiPAP / VNI-PS & BiPAP \\
\hline Interface & Máscara facial o nasal & Máscara facial o nasal \\
\hline Pacientes & $\begin{array}{l}97 \text { pacientes ventilados que toleraron una } \\
\text { prueba de respiración espontánea y fueron } \\
\text { considerados de alto riesgo de fracaso en el } \\
\text { destete }\end{array}$ & $\begin{array}{l}162 \text { pacientes ventilados que toleraron una } \\
\text { prueba de respiración espontánea y fueron } \\
\text { considerados de alto riesgo de fracaso en el } \\
\text { destete }\end{array}$ \\
\hline Intervención & $\begin{array}{l}\text { VNI: } 48 \\
\text { CT: } 49\end{array}$ & $\begin{array}{l}\text { VNI: } 79 \\
\text { CT: } 83\end{array}$ \\
\hline $\begin{array}{l}\text { Falla respiratoria } \\
\text { post extubación }\end{array}$ & $\begin{array}{l}\text { VNI: } 8,3 \% \\
\text { CT: } 24,5 \% \\
p=0,027\end{array}$ & $\begin{array}{l}\text { VNI: } 16 \% \\
\text { CT: } 33 \% \\
p=0,029\end{array}$ \\
\hline Estadía en UCI & $\begin{array}{l}\text { VNI: } 8,9 \pm 5,7 \mathrm{~d} \\
\text { CT: } 11,6 \pm 14,9 \mathrm{~d} \\
\text { NS }\end{array}$ & $\begin{array}{l}\text { VNI: } 11 \pm 8 \mathrm{~d} \\
\text { CT: } 13 \pm 11 \mathrm{~d} \\
\text { NS }\end{array}$ \\
\hline Estadía en el hospital & $\begin{array}{l}\text { VNI: } 23,3 \pm 16,4 \mathrm{~d} \\
\text { CT: } 25,5 \pm 21,4 \mathrm{~d} \\
\text { NS }\end{array}$ & $\begin{array}{l}\text { VNI: } 30 \pm 23 \mathrm{~d} \\
\text { CT: } 29 \pm 18 \mathrm{~d} \\
\text { NS }\end{array}$ \\
\hline Mortalidad en la UCI & $\begin{array}{l}\text { VNI: } 6,2 \% \\
\text { CT: } 18,4 \% \\
p<0,001\end{array}$ & $\begin{array}{l}\text { VNI: } 3 \% \\
\text { CT: } 14 \% \\
p=0,015\end{array}$ \\
\hline $\begin{array}{l}\text { Mortalidad en el } \\
\text { hospital }\end{array}$ & $\begin{array}{l}\text { VNI: } 12 \% \\
\text { CT: } 18 \% \\
\text { NS }\end{array}$ & $\begin{array}{l}\text { VNI: } 16 \% \\
\text { CT: } 23 \% \\
\text { NS }\end{array}$ \\
\hline
\end{tabular}

VNI: ventilación no invasiva, CT: grupo control, VNI-PS: ventilación no invasiva-presión de soporte, BiPAP: presión positiva en la vía aérea de dos niveles. 
dos días y 49 pacientes recibieron tratamiento médico estándar. Los pacientes manejados con VNI tuvieron menor incidencia de reintubación $(8,3 \%$ vs $24,5 \%$; $\mathrm{p}=0,027)$ y mortalidad en la UCI.

En otro ensayo clínico randomizado, 162 pacientes de alto riesgo ventilados durante más de dos días y que toleraron una prueba de respiración espontánea en tubo $\mathrm{T}$ fueron asignados a recibir VNI continua durante 24 horas o tratamiento médico estándar ${ }^{16}$. Los factores de riesgo de falla respiratoria post extubación fueron los siguientes: edad mayor de 65 años, insuficiencia cardíaca, o APACHE II > 12 el día de la extubación. Setenta y nueve pacientes recibieron VNI y 83 recibieron tratamiento convencional. La VNI redujo el riesgo de falla respiratoria post extubación ( $16 \%$ vs $33 \%$; $\mathrm{p}=0,029)$, disminuyó el riesgo de reintubación (11\% vs 22\%) y la mortalidad en la UCI ( $3 \%$ vs $14 \%$; $\mathrm{p}=0,015)$. La sobrevida en el hospital y en el seguimiento a 90 días fueron similares en ambos grupos. En el análisis de subgrupos, la VNI disminuyó significativamente el riesgo de muerte en la UCI, el hospital y en el seguimiento a 90 días en los pacientes con falla respiratoria hipercápnica durante la prueba de respiración espontánea en tubo $\mathrm{T}$.

Ambos estudios, los cuales adoptaron criterios similares para definir las categorías de riesgo y tuvieron diseños comparables, han mostrado que la aplicación de VNI inmediatamente después de la extubación disminuye el riesgo de reintubación y la mortalidad en la $\mathrm{UCI}^{15,16}$. Los pacientes con enfermedad pulmonar obstructiva crónica y falla respiratoria hipercápnica son los que parecen mostrar el mayor beneficio.

c) Disminuir el riesgo de fracaso en pacientes con falla respiratoria progresiva después de la extubación. El fracaso de la extubación se asocia a una alta morbimortalidad. Se ha sugerido el uso de VNI en un intento de evitar la reintubación en pacientes que muestran signos incipientes o incluso falla respiratoria establecida después de la extubación (Tabla 3). A pesar de los resultados promisorios de un estudio casocontrol en pacientes hipercápnicos ${ }^{17}$, un ensayo controlado no encontró que la VNI disminuyera

Tabla 3. Estudios clínicos controlados que examinan la eficacia de la ventilación no invasiva en pacientes con falla respiratoria post extubación

\begin{tabular}{|c|c|c|}
\hline Efecto de VNI & Keenan $^{18}$ & Esteban $^{19}$ \\
\hline Publicación & 2002 & 2004 \\
\hline Modalidad & BiPAP & VNI-PS \\
\hline Interface & Máscara facial & Máscara facial \\
\hline Pacientes & $\begin{array}{l}81 \text { pacientes ventilados, con enfermedad } \\
\text { cardíaca o pulmonar, que desarrollaron } \\
\text { dificultad respiratoria en las } 48 \text { horas post } \\
\text { extubación }\end{array}$ & $\begin{array}{l}221 \text { pacientes ventilados que desarrollaron } \\
\text { dificultad respiratoria en las } 48 \text { horas post } \\
\text { extubación }\end{array}$ \\
\hline Intervención & $\begin{array}{l}\text { VNI: } 39 \\
\text { CT: } 42\end{array}$ & $\begin{array}{l}\text { VNI: } 114 \\
\text { CT: } 107\end{array}$ \\
\hline Tasa de intubación & $\begin{array}{l}\text { VNI: } 72 \% \\
\text { CT: } 69 \% \\
\text { NS }\end{array}$ & $\begin{array}{l}\text { VNI: } 48 \% \\
\text { CT: } 48 \% \\
\text { NS }\end{array}$ \\
\hline Estadía en UCI & $\begin{array}{l}\text { VNI: } 15,1 \pm 10,9 \mathrm{~d} \\
\text { CT: } 19,4 \pm 25,0 \mathrm{~d} \\
\text { NS }\end{array}$ & $\begin{array}{l}\text { VNI: } 18 \mathrm{~d}(\mathrm{R}: 11-30) \\
\text { CT: } 18 \mathrm{~d}(\mathrm{R}: 11-26) \\
\text { NS }\end{array}$ \\
\hline Estadía en el hospital & $\begin{array}{l}\text { VNI: } 32,2 \pm 25,4 \mathrm{~d} \\
\text { CT: } 29,8 \pm 28,4 \mathrm{~d} \\
\text { NS }\end{array}$ & \\
\hline Mortalidad en la UCI & $\begin{array}{l}\text { VNI: } 15 \% \\
\text { CT: } 24 \% \\
\text { NS }\end{array}$ & $\begin{array}{l}\text { VNI: } 25 \% \\
\text { CT: } 14 \% \\
p=0,048\end{array}$ \\
\hline $\begin{array}{l}\text { Mortalidad en el } \\
\text { hospital }\end{array}$ & $\begin{array}{l}\text { VNI: } 31 \% \\
\text { CT: } 31 \% \\
\text { NS }\end{array}$ & \\
\hline
\end{tabular}

VNI: ventilación no invasiva, CT: grupo control, BiPAP: presión positiva en la vía aérea de dos niveles, VNI-PS: ventilación no invasiva-presión de soporte. 
la tasa de reintubación (RR: 1,04; IC95\%: 0,781,38) o la mortalidad en el hospital (RR: 0,99; IC95\%: 0,52-1,91) en los pacientes que desarrollaron insuficiencia respiratoria aguda dentro de las 48 horas siguientes a la extubación ${ }^{18}$. El estudio de Keenan y cols, fue realizado en un único centro, enrolaron a 81 pacientes con enfermedad cardíaca o pulmonar crónica y, después del primer año, los pacientes con EPOC exacerbado fueron excluidos debido a razones éticas (este grupo claramente se beneficia con la VNI). En un estudio multicéntrico, en el que la VNI se inició tan pronto como los pacientes presentaron signos de dificultad respiratoria post extubación, Esteban y cols, no encontraron diferencias significativas en la tasa de reintubación (RR: 0,99; IC95\%: 0,76-1,30), en cambio observaron un aumento de la mortalidad en la UCI en los pacientes ventilados no invasivamente (RR: 1,78; IC95\%: 1,03-3,20; $p=0,048$ ), lo cual fue atribuido a que en estos pacientes se retrasó la reintubación ${ }^{19}$. En este estudio se enrolaron 221 pacientes que desarrollaron falla respiratoria aguda después de la extubación, y sólo $10 \%$ de los pacientes tenía EPOC. Estos hallazgos no apoyan, ciertamente, el uso rutinario de VNI en el manejo de los pacientes con falla respiratoria progresiva después de la extubación.

\section{Efecto potencial}

La VNI facilitaría la desconexión del ventilador en pacientes con destete difícil, disminuyendo el tiempo de permanencia en VM y en el hospital, las complicaciones infecciosas y mortalidad. El efecto beneficioso se ha observado básicamente en pacientes con EPOC y falla respiratoria hipercápnica, aún faltan estudios que avalen su empleo rutinario en el proceso de desconexión del VM de otras causas de falla respiratoria, tales como síndrome de dificultad respiratoria aguda, complicaciones post cirugía o falla cardiovascular.

Los estudios clínicos han mostrado beneficios de la VNI como intervención temprana adicionada al tratamiento médico habitual en pacientes de riesgo elevado para evitar la falla respiratoria aguda post extubación. En cambio, los estudios clínicos no han demostrado su eficacia en pacientes con falla respiratoria establecida después de la extubación e incluso pudiera ser deletérea.

\section{Evaluación del costo/beneficio}

De acuerdo a la información disponible, la VNI aplicada en el destete difícil de pacientes con EPOC exacerbado y falla respiratoria aguda hipercápnica sería una medida costo/efectiva: disminuye el tiempo de permanencia conectado al ventilador, la estadía en la UCI y el hospital, las complicaciones infecciosas asociadas, y reduce la mortalidad.

\section{Grado de recomendación}

La ventilación no invasiva debe ser considerada una terapia de primera línea en el manejo de pacientes con EPOC exacerbado, falla respiratoria hipercápnica y destete difícil del ventilador mecánico (recomendación moderada sustentada en evidencia de buena calidad).

\section{Bibliografía}

1.- TORRES A, AZNAR R, GATELL J M, JIMÉNEZ P, GONZÁLEZ J, FERRER A, et al. Incidence, risk, and prognosis factors of nosocomial pneumonia in mechanically ventilated patients. Am Rev Respir Dis 1990; 142: 523-8.

2.- ESTEBAN A, ALIA I, IBÁÑEZ J, BENITO S, TOBIN M J; the Spanish Lung Failure Collaborative Group. Modes of mechanical ventilation and weaning. A national survey of Spanish hospitals. Chest 1994; 106 : 1188-93.

3.- NAVA S, RUBINI F, ZANOTTI E, AMBROSINO N, BRUSCHI C, VITACCA M, et al. Survival and prediction of successful ventilator weaning in COPD patients requiring mechanical ventilation for more than 21 days. Eur Respir J 1994; 7: 1645-52.

4.- TORRES A, GATELL J M, AZNAR E, EL-EBIARY M, PUIG DE LA BELLACASA J, GONZÁLEZ J, et al. Re-intubation increases the risk of nosocomial pneumonia in patients needing mechanical ventilation. Am J Respir Crit Care Med 1995; 152: 137-41.

5.- International Consensus Conferences in Intensive Care Medicine: Noninvasive positive pressure ventilation in acute respiratory failure. Am J Respir Crit Care Med 2001; 163: 283-91.

6.- NAVA S, NAVALESI P, CARLUCCI A. Non-invasive ventilation. Minerva Anestesiol 2008; 74: 1-6.

7.- FERRER M. Non-invasive ventilation in the weaning process. Minerva Anestesiol 2008; 74: 311-4.

8.- NAVA S, AMBROSINO N, CLINI E, PRATO M, ORLANDO G, VITACCA M, et al. Noninvasive mechanical ventilation in the weaning of patients with respiratory failure due to chronic obstructive pulmonary disease. A randomized, controlled trial. Ann Intern Med 1998; 128: 721-8.

9.- GIRAULT C, DAUDENTHUN I, CHEVRON V, TAMION F, LEROY J, BONMARCHAND G. Noninvasive ventilation as a systematic extubation and weaning technique in acute-on-chronic respiratory failure. A prospective, randomized controlled study. Am J Respir Crit Care Med 1999; 160: 86-92.

10.- CHEN J, QIU D, TAO D. Time for extubation and sequential noninvasive mechanical ventilation in COPD patients with exacerbated respiratory failure who received invasive ventilation. Zhonghua Jie $\mathrm{He} \mathrm{He} \mathrm{Hu}$ Xi Za Zhi 2001; 24: 99-100.

11.- FERRER M, ESQUINAS A, ARANCIBIA F, THOMAS BAUER T, GONZÁLEZ G, CARRILLO A, et al. 
Noninvasive ventilation during persistent weaning failure. A randomized controlled trial. Am J Respir Crit Care Med 2003; 168: 70-6.

12.- HILL N S, LIN D, LEVY M, et al. Noninvasive positive pressure ventilation (NPPV) to facilitate extubation after acute respiratory failure: a feasibility study. Am J Respir Crit Care Med 2000; 161: A263 (abstract).

13.- BURNS K E, ADHIKARI N K J, MEADE M O. A meta-analysis of noninvasive weaning to facilitate liberation from mechanical ventilation. Can J Anesth 2006; 53: 305-15.

14.- EPSTEIN S K, CIUBOTARU R L. Independent effects of etiology of failure and time of reintubation on outcome for patients failing extubation. Am J Respir Crit Care Med 1998; 158: 489-93.

15.- NAVA S, GREGORETTI C, FANFULLA F, SQUADRONE E, GRASSI M, CARLUCCI A, et al. Noninvasive ventilation to prevent respiratory failure after extubation in high risk patients. Crit Care Med
2005; 33: 2465-70.

16.- FERRER M, VALENCIA M, NICOLAS J M, BERNADICH O, BADIA J R, TORRES A. Early noninvasive ventilation averts extubation failure in patients at risk. A randomized trial. Am J Respir Crit Care Med 2006; 173: 164-70.

17.- HILBERT G, GRUSON D, PORTEL L, GBIKPIBENISSAN G, CARDINAUD J P. Noninvasive pressure support ventilation in COPD patients with postextubation hypercapnic respiratory insufficiency. Eur Respir J 1998; 11: 1349-53.

18.- KEENAN S P, POWERS C, MCCORMACK D G, BLOCK G. Noninvasive positive-pressure ventilation for postextubation respiratory distress. A randomized controlled trial. JAMA 2002; 287: 3238-44.

19.- ESTEBAN A, FRUTOS-VIVAR F, FERGUSON N D, ARABI Y, APEZTEGUÍA C, GONZÁLEZ M, et al. Noninvasive positive-pressure ventilation for respiratory failure after extubation. N Engl J Med 2004; 350: $2452-60$. 